\title{
Regulation of yeast RPL22B splicing depends on intact pre-mRNA context of the intron
}

\author{
Kateřina Abrhámová and Petr Folk* \\ Department of Cell Biology, Faculty of Science, Charles University, Viničná 7, \\ 12800 Praha, Czech Republic
}

*Corresponding author: Email: petr.folk@natur.cuni.cz; Tel.: +420 221951765

Keywords: intergenic regulation; pre-mRNA splicing; RNA structure; Saccharomyces cerevisiae; ribosomal protein genes

\section{Abstract}

Yeast RPL22A and RPL22B genes form an intergenic regulatory loop modulating the ratio of paralogous transcripts in response to changing levels of proteins. Gabunilas and Chanfreau (Gabunilas and Chanfreau, PLoS Genet 12, e1005999, 2016) and our group (Abrhámová et al., PLoS ONE 13, e0190685, 2018) described that Rpl22 proteins bound to the divergent introns of RPL22 paralogs and inhibited splicing in dosage dependent manner. Here, we continued to study the splicing regulation in more detail and designed constructs for in vivo analyses of splicing efficiency. We also tested Rpl22 binding to RPL22B intron in three-hybrid system. We were able to confirm the findings reported originally by Gabunilas and Chanfreau on the importance of a stem loop structure within the RPL22B intron. Mutations which lowered the stability of the structure abolished Rpl22-mediated inhibition. In contrast, we were not able to confirm the sequence specificity with respect to either Rpl22 binding or splicing inhibition within this region, which they reported. We contradict their results that the 'RNA internal loop' of RPL22Bi (nt 178CCCU181 and 221UGAA224) is crucial for mediating the Rpl22 effects. We assume that this discrepancy reflects the difference in constructs, as the reporters used by Gabunilas and Chanfreau lacked the alternative 5' splice site as well as surrounding exons. Our own comparison confirms that deleting the sequence spanning alternative 5' splice site lowers splicing efficiency, hinting to possible disturbances of the regulatory mechanism. We argue that the structural context of the 'regulatory element' may reach across the intron or into the surrounding sequences, similarly to what was found previously for other genes, such as RPL30. Apparently, more detailed analyses are needed to discern this intriguing example of splicing regulation. 


\section{Introduction}

Ribosomal proteins (RPs) constitute structural and regulatory components of ribosomes and as such remain highly conserved during evolution. Despite this constraint, some RPs acquired extra ribosomal functions by binding to RNAs other than rRNAs, mostly with impact on gene expression (Warner and McIntosh, 2009). The pre-mRNA molecules, their introns and untranslated regions in particular, can thus be viewed as evolving structures, which gained affinity for pre-existing RPs (Fahl et al., 2015). As it was found, Rpl26 bound p53 mRNA through its 5' untranslated region (5'UTR), which stimulated translation and thus contributed to the regulation of DNA-damage response (Takagi et al., 2005). Rpl13a, upon IFN- $\gamma$ activated phosphorylation, was released from ribosomes and translationally inhibited several groups of mRNAs as part of the GAIT system, which directed transcript selective translational control in myeloid cells (Arif et al., 2018; Mukhopadhyay et al., 2008).

While some RPs are unique, some RP coding genes (RPGs) underwent duplication and exist as paralog pairs. In yeast, which experienced whole genome duplication followed by loss of duplicates, RPGs, in their majority, retained their paralogs. These gene pairs were implicated in intergenic regulation (Parenteau et al., 2011). However, only a handful of such pairs were proven to regulate each other's expression, such as RPS14, RPS9, and RPL22 (Fewell and Woolford, 1999; Plocik and Guthrie, 2012; Petibon et al., 2016; Gabunilas and Chanfreau, 2016; Abrhámová et al., 2018). The incorporation of two different paralogs into ribosomes may give rise to ribosome heterogeneity, which became focus of recent attention. Proteomic and transcriptomic analyses found evidence for the existence of ribosomal subpopulations which differed in composition, interactomes, and perhaps mRNA specificity (Yadav et al., 2016; Shi et al., 2017; Segev and Gerst, 2018).

The propensity of RNA transcripts to form local structures of varying stability as well as interactions over long distances was studied for over 30 years (for reviews see Wan et al., 2011; Lin et al., 2016). More recently, structures begun to be examined in whole transcriptomes, using both chemical structure probing techniques as well as modelling approaches. Structures changed their parameters with cultivation conditions and upon stress, suggesting that RNA structural features might be of general significance for gene expression, including splicing and translation (Zhang et al., 2017; Rouskin et al., 2014; Kwok, 2016). Splicing sequences of 5' splice site (5'ss), branch point (BP), or 3' splice site (3'ss) can be incorporated into stems and thus blocked (Singh et al., 2006), or brought into proximity and made stronger (Lin et al., 2016; Rogic et al., 2008; Gahura et al., 2011). Exons can be looped out (Raker et al., 2009) or selected for alternative splicing through base- 
pairing interactions, such as in the case of multi-cluster mutually exclusive exons (Graveley, 2005; McManus and Graveley, 2011; Ivanov and Pervouchine, 2018).

$R P L 22$ paralogs represent an intriguing example of extra ribosomal function-gain and intergenic regulation throughout evolution. Extra ribosomal roles of Rpl22 paralogs were documented in yeast (see below), fruit fly, zebrafish, mice, and humans (Kearse et al., 2011; Mageeney et al., 2018; Zhang et al., 2017; O'Leary et al., 2013; Zhang et al., 2013). Human Rpl22 interacted with human telomerase RNA (Le et al., 2000) and was also sequestered, apparently in competition, by the Epstein-Barr virus (EBV)-encoded RNAs in EBV infected cells, possibly as part of the viral strategy to interfere with translation and growth (Fok, 2006; Houmani et al., 2009). Recently, Zhang and coworkers demonstrated that zebrafish Rpl22 and Rpl22-Like1 controlled morphogenesis during gastrulation. The proteins acted antagonistically to modulate splicing of Smad2 pre-mRNA, presumably in cooperation with HNRNP-A1 (Zhang et al., 2017).

In yeast, Rpl22A/B pair was shown to be part of oxidative stress response. Because $R P L 22 A$ but NOT RPL22B mRNA is UUG rich, and because translation efficiency of UUG rich transcripts was increased under stress, the translation of RPL22A became more efficient, leading to change in A/B ratio (Chan et al., 2012). The effect was mediated by the methyl methanesulfonate (MMS) sensitive tRNA methyltransferase 9 (Trm9) (Begley et al., 2004, 2007). Rpl22 was also shown to be required for IME1 mRNA translation and meiotic induction in $S$. cerevisiae, as $r p / 22 \Delta$ cells were unable to translate the IME1 transcript because of its atypical 5'UTR (Kim and Strich, 2016). On the level of splicing regulated gene expression, the feedback-loop formed by the $R P L 22 A / B$ pair coupled free $[A]+[B]$ fluctuations to changes in $[A] /[B]$. The protein products of either paralog bound to RPL22 introns, which caused moderate inhibition of the major paralog (RPL22A) and strong inhibition of the minor one (RPL22B) (Abrhámová et al., 2018). A/B changes were related to oxidative stress (see above), or disbalances in free RP concentration caused by exposures to MMS or Cd (Gabunilas and Chanfreau, 2016), supporting the interpretation that tuning the isoform ratio helps the cells to react to changing environment. What cellular process or machinery distinguishes the 19 amino acids difference between Rpl22A and Rpl22B remains unknown; the effects on splicing are apparently mediated by both $A$ and $B$ proteins (Abrhámová et al., 2018). Information on the interface between the intron and Rpl22, and between this complex and the spliceosome, is likewise limited. Gabunilas and Chanfreau postulated the intronic region between nt 153 and 225 (positions here and throughout the text are numbered relative to the first nucleotide of the RPL22B intron) as 'regulatory element' and found that it was responsible for protein binding (Fig3C, D in Gabunilas and Chanfreau, 2016). This is similar 
to our conclusions based on yeast three-hybrid assays (nt 165 to 236; see I2, Abrhámová et al., 2018). They also showed that a predicted stem forming between nt 182-188 and 214-220 is necessary for Rpl22-mediated regulation and can rescue splicing inhibition in vitro when added in trans as part of free RNA (nt 115-255) (Gabunilas and Chanfreau, 2016).

Functional relationship of a ribosomal protein to both rRNA and its own intronic RNA, as found in $R P L 22 A / B$, is interesting for both structural and evolutionary biology. We began to characterize the intron-Rpl22 interaction in more detail, using constructs for in vivo measurements of splicing efficiency and yeast three-hybrid system. Our data agree with the findings of Gabunilas and Chanfreau (Gabunilas and Chanfreau, 2016) in that disrupting a stem structure within the RPL22B intron abolishes Rpl22-mediated inhibition. However, we contradict their conclusion that a sequence element within the 'RNA internal loop' (RPL22Bi nt 178CCCU181 and 221UGAA224) is crucial for Rpl22 binding and splicing inhibition. We confirmed that the intron's response is dependent on the presence of intact alternative 5' splice site (ALT5'ss), which suggests that the inhibitory function of the intron involves not only the 182-188/214-220 stem, but also the 5' proximal region of the intron and potentially upstream parts of the transcript.

\section{Results}

We decided to study the properties of the RPL22 introns in more detail, using sensitive in vivo reporter assay and yeast three-hybrid analysis. Our aim is to decipher the mechanism which RPL22 derived RNA molecules use in the regulation of expression of their own genes.

We set out to design splicing constructs harboring RPL22B intron surrounded by its own 5'UTR and exon 1 and by 60 nt of exon 2 fused in frame with CUP1 reporter on the intron's 5' and 3' ends, respectively (see Fig1A). In this way, any structural and functional features of the pre-mRNA molecule between nt -56 and +381 (positions are numbered relative to the first nucleotide of the RPL22 intron) were preserved, including ALT5'ss (65GTTTGT). We introduced mutations in regions implicated in Rpl22 mediated regulation and binding and assessed their impact on splicing. We employed cells with both endogenous RPL22 paralogs deleted, which were harboring an expression plasmid, either empty or bearing RPL22A. We could thus test the splicing of $R P L 22 B$ in the absence or the presence of Rpl22A and directly compare the readouts. This setup allowed us to differentiate mutations which were (i) splicing inhibitory regardless of the presence or absence of Rpl22 protein from those which were (ii) splicing permissive in its presence - rendering the intron unresponsive 
to Rpl22-mediated inhibition. In addition, some mutations (iii) showed graded effect and some (iv) affected the relative usage of alternative splice site.

One example of splicing permissive mutation is the destabilization of a hypothetical stem between nt 182-188 and 214-220 of RPL22Bi (Fig2A, B). This stem can be predicted using both RNA fold and Mfold (Lorenz et al., 2011; Zuker, 2003). To facilitate comparison between the work of Gabunilas and Chanfreau and our own when referring to mutations, we adopted the denotations used by these authors (Fig2C). We found that deleting the nucleotides $214-220$ of the 3' arm of the 'lower distal stem' (LDS) turned the intron refractory to Rpl22A inhibition. To further confirm that LDS is important for the negative regulation of $R P L 22 B$ intron splicing, we prepared constructs coding for stems of similar predicted stability but with the LDS arms flipped (LDS'Flip') or scrambled (LDS'Scramble') to eliminate any nucleotide-specific effects. As shown in Fig2B, these constructs retained WT behavior. In contrast, mutations leading to decreased pairing probability within LDS (LDS ' $\Delta 3$ ', LDS 'Loose') led to splicing permissive outcome - the intron's responsiveness to Rpl22A was almost lost.

Targeting the ALT5'ss (deletion of nt 65-70) impaired splicing (Fig2A). We observed the accumulation of unspliced pre-mRNA even in the absence of the inhibitory protein in comparison with WT construct, where pre-mRNA was not detected. The mutant construct, however, still maintained some regulatory potential as the unspliced pre-mRNA signal was more pronounced in the presence of Rpl22A. The result showing that the deletion of ALT5'ss negatively affects splicing efficiency of RPL22Bi was already obtained by Chanfreau group (Fig5B in Kawashima et al., 2014; FigS2C in Gabunilas and Chanfreau, 2016).

In addition to LDS, Gabunilas and Chanfreau targeted several other parts of the structured region between nt 153 and 225 of RPL22Bi. Based on their results, the authors proposed a more detailed model of RPL22Bi-Rpl22A interaction (FigS4 and S5 in Gabunilas and Chanfreau, 2016). We were concerned by the fact that their constructs did not include the neighboring regions of the intron and, most importantly, contained the deletion of ALT5'ss, shown by both groups to change splicing of RPL22Bi. We therefore decided to implement the above mentioned modifications in our constructs and test their behavior under our conditions, using the entire RPL22B intron with ALT5'ss and the surrounding sequences. We prepared constructs DS Internal Loop CAUU (UGAA to CAUU mutation), US Internal Loop AAAC (CCCU to AAAC mutation), $\triangle$ Internal Loop (deletion of the entire 'RNA internal loop') as well as $\triangle$ DS Internal Loop and $\triangle$ US Internal Loop, where the 5' or 3' arm of the 'RNA internal loop' is deleted. We found that none of the mutations disrupted negative splicing regulation by Rpl22A because all the reporters reacted to the Rpl22A overexpression 
by increasing the amount of unspliced pre-mRNA to levels obtained in the WT reporter (Fig2B). In the uninhibited state, they were also spliced with the same efficiency as the WT intron; possible exception was $\Delta$ Internal Loop mutant, which showed slightly less efficient splicing.

Rpl22A binds to parts of RPL22i, as was shown by Gabunilas and Chanfreau as well as by us. While there is still no ultimate proof that the binding is direct and not mediated or aided by other molecules, it can be reproduced in yeast three-hybrid system using only fragments of the intron. Rpl22A binding is dependent on the presence of lysines at the protein's RNA-binding interface, as mutants where these lysines are substituted by glutamates are binding-negative in the three-hybrid system and unable to inhibit RPL22B splicing in vivo (Abrhámová et al, 2018). We used three-hybrid system to test Rpl22A binding to the mutant versions of RPL22Bi (Fig3). None of the mutations targeting the 'RNA internal loop' (Fig2D) impaired the ability of this part of intron to activate reporter gene synthesis in yeast three-hybrid system, implying that they were still compatible with Rpl22 binding.

\section{Discussion}

Important aspect of $R P L 22 A / B$ biology is the differentially regulated splicing of their pre-mRNAs. RPL22 introns evolved into regulatory elements which block pre-mRNA maturation at high Rpl22 concentration. We would like to understand the mechanism of inhibition as it may be of general significance for the splicing field.

We constructed splicing reporters for in vivo testing of mutant introns and begun to characterize the elements which are necessary and sufficient for the Rpl22-dependent regulation (Fig1A). We found that deletion of the ALT5'ss of RPL22Bi impairs splicing efficiency at the major site (Fig2A). In previously published studies, we and others have shown that splicing in vivo uses alternative $5^{\prime}$ as well as $3^{\prime}$ splice sites, albeit at low frequencies (Abrhámová et al., 2018; Kawashima et al., 2014; Schreiber et al., 2015; Gould et al., 2016; Aslanzadeh et al., 2018). While the alternative site does not lead to a functional product, it may be part of the spliceosome assembly process, e.g., as an adjunct assembly landing platform (Libri et al., 2000; Spingola and Ares, 2000). In all our constructs, we retain the ALT5'ss and also include intron proximal sequences.

The inclusion of intron-surrounding sequences and the presence of intact ALT5'ss represent important differences in approach between Gabunilas and Chanfreau and our lab. On the beginning of their search for the region responsible for the regulation of RPL22Bi 
splicing, Gabunilas and Chanfreau prepared deletions lacking the first ( $\Delta 7-152)$ or the second ( $\Delta 153-297)$ half of the sequence between 5 'ss and BP. The construct $\Delta 7-152$ retained the capacity to inhibit splicing in the presence of RPL22A, but it caused increased accumulation of unspliced transcript in $r p / 22 a \Delta$ cells. The construct $\Delta 153-297$, on the other hand, gave less clear result and showed increased usage of ALT5'ss (FigS2A in Gabunilas and Chanfreau, 2016). At this point, the authors decided to delete the ALT5'ss (FigS2C in Gabunilas and Chanfreau, 2016). Their own results show, however, that $\triangle A L T 5$ 'ss-intron is splicing-impaired, both in the presence and absence of Rpl22A (compare lanes 1 to 3 and 2 to 4 in FigS2C in Gabunilas and Chanfreau, 2016). The $\Delta 153-297 \Delta$ ALT5'ss construct showed relieved inhibition in WT cells, but unspliced pre-mRNA continued to be increased in the absence of Rpl22A. Strikingly, the deletion of ALT5'ss had opposite impact on splicing efficiency of WT intron (inhibition of splicing in both WT and rp/22a $\Delta$ cells) in comparison with its truncated version ( $\Delta 153-297$; improvement of splicing). We believe that their as well as our deletion constructs show that the region between 5'ss and BP, including the ALT5'ss, is required for full regulatory capacity of the intron. Notably, mutations or ablations of intronadjacent exon sequences were shown to impact splicing efficiency (Crotti et al., 2007; Chanfreau et al., 1999). It was also found that splicing of RPS9 was regulated by the interplay between an intronic structure and 3' untranslated region (Petibon et al., 2016). The results on RPL22Bi, where both ALT5'ss deletion and other manipulations are present simultaneously, may thus be difficult to interpret unless we understand the structure-function relationships in detail.

We assume that the above differences may be the reason why our results on the mutations of the 'RNA internal loop' contradict the conclusions of Gabunilas and Chanfreau. We found that the constructs US Internal Loop AAAC and DS Internal Loop CAUU both maintained their regulatory potential (Fig2D), as did $\Delta$ DS Internal Loop and $\Delta$ US Internal Loop. Only $\Delta$ Internal Loop construct showed slight decrease of splicing efficiency in the absence of Rpl22, but even this construct inhibited splicing in response to Rpl22 overexpression. Gabunilas and Chanfreau first tested $\Delta 191-211$ UUCG $\Delta$ Internal Loop mutant, which was in their assay more splicing permissive than $\Delta 191-211$ UUCG mutant, suggesting to them that the internal loop nucleotides are important for splicing regulation. However, these constructs lacked the apical portion of the stem between nt 191 and 211, which obviously made them fold differently from the full-length stem, irrespective of the internal loop deletion. Second, they found that DS Internal Loop CAUU manipulation is more splicing permissive than US Internal Loop AAAC (FigS5B in Gabunilas and Chanfreau, 2016), which led them to propose that the sequence GUAA (mutated in CAUU) is crucial for 
mediating the effect of Rpl22A. Unfortunately, Gabunilas and Chanfreau did not include comparisons between WT, rp/22a $\Delta$ and Rpl22A overexpressing cells (Fig3, FigS4 and S5 in Gabunilas and Chanfreau, 2016). This makes it difficult to judge on the capacity of the constructs to regulate in response to changing Rpl22A concentration.

To complement our findings in Fig3C, we tested all our mutants (see also above) for their capacity to bind Rpl22 in three-hybrid assay. Indeed, none of the mutations hampered Rpl22A binding; the binding strength, based on the ability of reporter cells to grown on 15mM 3-AT, was identical to WT. This further supports our assumption that the mutations of the 'RNA internal loop' nucleotides, including its DS arm GUAA (nt 178CCCU181 and 221UGAA224), do not disturb Rpl22A mediated inhibition.

The regulatory mechanism of the intron may encompass not only the stem between nt 182-188 and 214-220 but also 5' upstream parts of the intron including the ALT5'ss. Splicing was shown to be modulated from regions outside of the introns themselves through (i) SR and other proteins binding outside of introns to exonic splicing silencers/ enhancers (for review see Lee and Rio, 2015), and (ii) RNA structures involving but not limited to exons (AbuQattam et al., 2018). In one of the well documented examples from S. cerevisiae, ribosomal Rpl30 protein binds to a stem-loop structure formed within the first exon and the 5' end of the intron of RPL30 transcript. The binding of the protein was shown to modulate both splicing (Eng and Warner, 1991; Vilardell and Warner, 1994) and translation, as the stemloop can apparently form even after splicing (Eng and Warner, 1991; Mao, 1999; Vilardell and Warner, 1994; Vilardell et al., 2000). The effects of Rpl30 on RPL30 splicing, which are brought about by RNA structure involving both intron and exon, can be taken as an illustration that the mechanism regulating splicing can be understood only by taking into account the context of the whole gene.

The results presented in this communication illustrate the requirement of ALT5'ss for $R P L 22 B i$ regulation. At the same time, they do not support the role of 'RNA internal loop' as the crucial interface required for Rpl22-triggered mechanism. Our results agree with Gabunilas and Chanfreau on the role of the 'lower distal stem', despite the differences in constructs. We assume that the structural disturbance (of making the sequences of LDS pairing-incompatible) is so substantial that it melts the RNA structure, eliminating both Rpl22A binding as well as any potential inhibitory effects. The involvement of ALT5'ss for $R P L 22 B i$ regulation would also explain the failure of Gabunilas and Chanfreau to show that their 'regulatory element' can function in an unrelated intron (RPS21A; Gabunilas and Chanfreau, 2016). 


\section{Methods}

\section{Yeast strains and cultivation methods}

Yeast strains were transformed using lithium acetate method (Gietz and Schiestl, 2007). Plasmids used in this study are listed in Table 1. rp/22a $\Delta$ rp/22b $\Delta$ strain was prepared as described previously (Abrhámová et al., 2018). For splicing analysis, yeast cells were inoculated from overnight grown pre-cultures and let to grow for two generations in synthetic medium without uracil and histidine. Yeast three-hybrid system testing was done essentially as described previously (Abrhámová et al., 2018).

\section{Splicing analyses}

RNA isolation and reverse transcription were performed as described previously (Abrhámová et al., 2018). Semiquantitative PCR was run in $25 \mu$ l-reactions with $5 \mu$ of 50 times diluted cDNA as template and with primers listed in Table2 for 25 to 28 cycles. The whole sample was loaded on $2.5 \%$ agarose gel and DNA was stained by ethidium bromide. Pictures were taken by Panasonic DMC-FZ7 camera, processed in GNU Image Manipulation Program 2.10.6 (https://www.gimp.org) and assembled in Inkscape 0.92 .3 (https://www.inkscape.org).

\section{Plasmid preparation}

$R P L 22 B$ intron mutants were synthetized by GeneArt (Thermo Fisher Scientific) and swapped using BamHI and EcoRI restriction sites with WT version of RPL22B in p423GPDRPL22B-CUP1. For three-hybrid testing, mutated versions of RPL22B were amplified from GeneArt DNA Strings with primers specific for each mutant (listed in Table 2) and cloned in Sphl site in p3HR2 vector. Cloning outcome was verified by restriction analysis and sequencing. 
Table 1. List of plasmids used in this study.

\begin{tabular}{|c|c|c|}
\hline Plasmid name & Insert & Source \\
\hline pVTU260 & - & (Melcher, 2000) \\
\hline pVTU260-RPL22A $\Delta \mathrm{i}$ & $R P L 22 A \triangle i$ & (Abrhámová et al., 2018) \\
\hline pVTU260-RPL22B $\Delta \mathrm{i}$ & $R P L 22 B \triangle i$ & (Abrhámová et al., 2018) \\
\hline $\begin{array}{l}\text { p423GPD-RPL22B- } \\
\text { CUP1 }\end{array}$ & $R P L 22 B$ - CUP1 fusion & (Abrhámová et al., 2018) \\
\hline $\begin{array}{l}\text { p423GPD- } \\
\text { RPL22B_AAlt5'ss- } \\
\text { CUP1 }\end{array}$ & RPL22B - CUP1 fusion with deletion of ALT5'ss & this study \\
\hline $\begin{array}{l}\text { p423GPD- } \\
\text { RPL22B_AUS-CUP1 }\end{array}$ & $\begin{array}{l}\text { RPL22B - CUP1 fusion with deletion of US } \\
\text { internal RNA Loop CCCU }\end{array}$ & this study \\
\hline $\begin{array}{l}\text { p423GPD- } \\
\text { RPL22B } \triangle \text { DS-CUP1 }\end{array}$ & $\begin{array}{l}R P L 22 B \text { - CUP1 fusion with deletion of DS } \\
\text { internal RNA Loop UGAA }\end{array}$ & this study \\
\hline $\begin{array}{l}\text { p423GPD- } \\
\text { RPL22B_AUSDS- } \\
\text { CUP1 }\end{array}$ & $\begin{array}{l}R P L 22 B \text { - CUP1 fusion with deletion of US } \\
\text { internal RNA Loop CCCU and of DS internal RNA } \\
\text { Loop UGAA }\end{array}$ & this study \\
\hline $\begin{array}{l}\text { p423GPD- } \\
\text { RPL22B DSmut-CUP1 }\end{array}$ & $\begin{array}{l}\text { RPL22B - CUP1 fusion with mutation of DS } \\
\text { internal RNA Loop UGAA to CAUU }\end{array}$ & this study \\
\hline $\begin{array}{l}\text { p423GPD/ } \\
\text { L22Bintron_USmut- } \\
\text { CUP1 }\end{array}$ & $\begin{array}{l}\text { RPL22B - CUP1 fusion with mutation of US } \\
\text { internal RNA Loop CCCU to AAAC }\end{array}$ & this study \\
\hline $\begin{array}{l}\text { p423GPD/ } \\
\text { L22Bintron } \triangle 3 \text { '-CUP1 }\end{array}$ & $\begin{array}{l}\text { RPL22B - CUP1 fusion with deletion of nt 214- } \\
220\end{array}$ & this study \\
\hline рАCT2 & - & (SenGupta et al., 1996) \\
\hline p3HR2 & - & (Stumpf et al., 2008) \\
\hline pIIIA/IRE-MS2 & IRE-MS2 & (SenGupta et al., 1996) \\
\hline pAD-IRP & IRP & (SenGupta et al., 1996) \\
\hline pACT2-RPL22A $\triangle \mathrm{i}$ & RPL22A $\triangle i$ with activation domain & (Abrhámová et al., 2018) \\
\hline p3HR2-I2 & $R P L 22 B$ intron nt 165 to 236 & (Abrhámová et al., 2018) \\
\hline p3HR2/AUS_I2 & $\begin{array}{l}R P L 22 B \text { intron nt } 165 \text { to } 236 \text { with deletion of US } \\
\text { internal RNA Loop CCCU }\end{array}$ & this study \\
\hline p3HR2/DSmut_I2 & $\begin{array}{l}R P L 22 B \text { intron nt } 165 \text { to } 236 \text { with mutation of DS } \\
\text { internal RNA Loop UGAA to CAUU }\end{array}$ & this study \\
\hline p3HR2/USmut_I2 & $\begin{array}{l}R P L 22 B \text { intron nt } 165 \text { to } 236 \text { with mutation of US } \\
\text { internal RNA Loop CCCU to AAAC }\end{array}$ & this study \\
\hline p3HR2/ADS_12 & $\begin{array}{l}R P L 22 B \text { intron nt } 165 \text { to } 236 \text { with deletion of DS } \\
\text { internal RNA Loop UGAA }\end{array}$ & this study \\
\hline p3HR2/AUSDS_I2 & $\begin{array}{l}R P L 22 B \text { intron nt } 165 \text { to } 236 \text { with deletion of US } \\
\text { internal RNA Loop CCCU and of DS internal RNA } \\
\text { Loop UGAA }\end{array}$ & this study \\
\hline
\end{tabular}


Table 2. List of primers used in this study.

\begin{tabular}{|l|l|l|}
\hline Primer & 'Sequence 5 $^{\prime} \rightarrow \mathbf{3}^{\prime}$ & Used for \\
\hline MO11 & GTTGGATCCGTAGCACAGTAAACC & RPL22B_in_p423GPD-CUP1_fw \\
\hline MO12 & CTGGAATTCAGTTGGAGAGGAAACG & RPL22B_in_p423GPD-CUP1_rev \\
\hline FN22 & ATATGCATGCTTTAAAAATTCACCCCTTATAGC & RPL22B_wt_in_p3HR2_fw \\
\hline FN23 & ACATGCATGCATTAACAATCACTTCATATAGCTAG & RPL22B_wt_in_p3HR2_rev \\
\hline KA189 & ACATGCATGCATTAACAATCACTATAGCTAGAGAA & RPL22B_deltaDS_in_p3HR2_rev \\
\hline KA180 & ATATGCATGCTTTAAAAATTCACTATAGCTTTA & RPL22B_deltaUS_in_p3HR2_fw \\
\hline KA181 & ACATGCATGCATTAACAATCACAATGTATAGCTAG & RPL22B_DSmut_in_p3HR2_rev \\
\hline KA182 & ATATGCATGCTTTAAAAATTCACAAACTATAGC & RPL22B_USmut_in_p3HR2_fw \\
\hline KA163 & GTAGCACAGTAAACCTATATCGTTTC & PCR_5'UTR_L22B_fw \\
\hline OG05 & GGCATTGGCACTCATGACCTTC & PCR_L22B_CUP1_rev \\
\hline KA104 & CTCAAAGCTGGCCAGTAGAA & PCR_SPT15_fw \\
\hline KA105 & CGTCACACGAACCGACAATA & PCR_SPT15_rev \\
\hline OG67 & CAAGGTAGTTCTGGGTCCTTAG & PCR_SCR1_fw \\
\hline OG68 & GACTGATATGTGCTATCCCG & PCR_SCR1_rev \\
\hline
\end{tabular}

\section{Acknowledgments}

We are grateful to Eva Krellerová for technical assistance. This work was supported by Charles University PROGRES program Q43 and UNCE 204013. 


\section{References}

Abrhámová, K., Nemčko, F., Libus, J., Převorovský, M., Hálová, M., Půta, F., and Folk, P. (2018). Introns provide a platform for intergenic regulatory feedback of RPL22 paralogs in yeast. PLoS ONE 13, e0190685.

AbuQattam, A., Serrano-Quílez, J., Rodríguez-Navarro, S., and Gallego, J. (2018). An exon three-way junction structure modulates splicing and degradation of the SUS1 yeast premRNA. Biochim. Biophys. Acta BBA - Gene Regul. Mech. 1861, 673-686.

Arif, A., Yao, P., Terenzi, F., Jia, J., Ray, P.S., and Fox, P.L. (2018). The GAIT translational control system: GAIT system. Wiley Interdiscip. Rev. RNA 9, e1441.

Aslanzadeh, V., Huang, Y., Sanguinetti, G., and Beggs, J.D. (2018). Transcription rate strongly affects splicing fidelity and cotranscriptionality in budding yeast. Genome Res. 28, 203-213.

Begley, T.J., Rosenbach, A.S., Ideker, T., and Samson, L.D. (2004). Hot Spots for Modulating Toxicity Identified by Genomic Phenotyping and Localization Mapping. Mol. Cell 16, 117-125.

Begley, U., Dyavaiah, M., Patil, A., Rooney, J.P., DiRenzo, D., Young, C.M., Conklin, D.S., Zitomer, R.S., and Begley, T.J. (2007). Trm9-Catalyzed tRNA Modifications Link Translation to the DNA Damage Response. Mol. Cell 28, 860-870.

Chan, C.T.Y., Pang, Y.L.J., Deng, W., Babu, I.R., Dyavaiah, M., Begley, T.J., and Dedon, P.C. (2012). Reprogramming of tRNA modifications controls the oxidative stress response by codon-biased translation of proteins. Nat. Commun. 3, 937.

Chanfreau, G., Gouyette, C., Schwer, B., and Jacquier, A. (1999). Trans-complementation of the second step of pre-mRNA splicing by exogenous 5' exons. RNA 5, 876-882.

Crotti, L.B., Bacikova, D., and Horowitz, D.S. (2007). The Prp18 protein stabilizes the interaction of both exons with the U5 snRNA during the second step of pre-mRNA splicing. Genes Dev. 21, 1204-1216.

Eng, F.J., and Warner, J.R. (1991). Structural basis for the regulation of splicing of a yeast messenger RNA. Cell 65, 797-804.

Fahl, S.P., Wang, M., Zhang, Y., Duc, A.-C.E., and Wiest, D.L. (2015). Regulatory Roles of Rpl22 in Hematopoiesis: An Old Dog with New Tricks. Crit. Rev. Immunol. 35, 379-400.

Fewell, S.W., and Woolford, J.L. (1999). Ribosomal Protein S14 of Saccharomyces cerevisiae Regulates Its Expression by Binding to RPS14B Pre-mRNA and to 18S rRNA. Mol. Cell. Biol. 19, 826-834.

Fok, V. (2006). Multiple domains of EBER 1, an Epstein-Barr virus noncoding RNA, recruit human ribosomal protein L22. RNA 12, 872-882.

Gabunilas, J., and Chanfreau, G. (2016). Splicing-Mediated Autoregulation Modulates Rpl22p Expression in Saccharomyces cerevisiae. PLoS Genet 12, e1005999. 
Gahura, O., Hammann, C., Valentová, A., Půta, F., and Folk, P. (2011). Secondary structure is required for 3' splice site recognition in yeast. Nucleic Acids Res. 39, 9759-9767.

Gietz, R.D., and Schiestl, R.H. (2007). High-efficiency yeast transformation using the LiAc/SS carrier DNA/PEG method. Nat. Protoc. 2, 31-34.

Gould, G.M., Paggi, J.M., Guo, Y., Phizicky, D.V., Zinshteyn, B., Wang, E.T., Gilbert, W.V., Gifford, D.K., and Burge, C.B. (2016). Identification of new branch points and unconventional introns in Saccharomyces cerevisiae. RNA 22, 1522-1534.

Graveley, B.R. (2005). Mutually Exclusive Splicing of the Insect Dscam Pre-mRNA Directed by Competing Intronic RNA Secondary Structures. Cell 123, 65-73.

Houmani, J.L., Davis, C.I., and Ruf, I.K. (2009). Growth-Promoting Properties of Epstein-Barr Virus EBER-1 RNA Correlate with Ribosomal Protein L22 Binding. J. Virol. 83, 9844-9853.

Ivanov, T., and Pervouchine, D. (2018). An Evolutionary Mechanism for the Generation of Competing RNA Structures Associated with Mutually Exclusive Exons. Genes 9, 356.

Kawashima, T., Douglass, S., Gabunilas, J., Pellegrini, M., and Chanfreau, G.F. (2014). Widespread Use of Non-productive Alternative Splice Sites in Saccharomyces cerevisiae. PLoS Genet. 10, e1004249.

Kearse, M.G., Chen, A.S., and Ware, V.C. (2011). Expression of ribosomal protein L22e family members in Drosophila melanogaster: rpL22-like is differentially expressed and alternatively spliced. Nucleic Acids Res. 39, 2701-2716.

Kerpedjiev, P., Hammer, S., and Hofacker, I.L. (2015). Forna (force-directed RNA): Simple and effective online RNA secondary structure diagrams. Bioinformatics 31, 3377-3379.

Kim, S.J., and Strich, R. (2016). Rpl22 is required for IME1 mRNA translation and meiotic induction in S. cerevisiae. Cell Div. 11.

Kwok, C.K. (2016). Dawn of the in vivo RNA structurome and interactome. Biochem. Soc. Trans. 44, 1395-1410.

Le, S., Sternglanz, R., and Greider, C.W. (2000). Identification of two RNA-binding proteins associated with human telomerase RNA. Mol. Biol. Cell 11, 999-1010.

Lee, Y., and Rio, D.C. (2015). Mechanisms and Regulation of Alternative Pre-mRNA Splicing. Annu. Rev. Biochem. 84, 291-323.

Libri, D., Lescure, A., and Rosbash, M. (2000). Splicing enhancement in the yeast rp51b intron. RNA 6, 352-368.

Lin, C.-L., Taggart, A.J., and Fairbrother, W.G. (2016). RNA structure in splicing: An evolutionary perspective. RNA Biol. 13, 766-771.

Lorenz, R., Bernhart, S.H., Zu Siederdissen, C.H., Tafer, H., Flamm, C., Stadler, P.F., and Hofacker, I.L. (2011). ViennaRNA Package 2.0. Algorithms Mol. Biol. 6, 26.

Mao, $H$. (1999). Assignment of the L30-mRNA complex using selective isotopic labeling and RNA mutants. Nucleic Acids Res. 27, 4059-4070. 
Mageeney, C.M., Kearse, M.G., Gershman, B.W., Pritchard, C.E., Colquhoun, J.M., and Ware, V.C. (2018). Functional interplay between ribosomal protein paralogues in the e RpL22 family in Drosophila melanogaster. Fly (Austin) 12, 143-163.

McManus, C.J., and Graveley, B.R. (2011). RNA structure and the mechanisms of alternative splicing. Curr. Opin. Genet. Dev. 21, 373-379.

Melcher, K. (2000). A Modular Set of Prokaryotic and Eukaryotic Expression Vectors. Anal. Biochem. 277, 109-120.

Mukhopadhyay, R., Ray, P.S., Arif, A., Brady, A.K., Kinter, M., and Fox, P.L. (2008). DAPKZIPK-L13a Axis Constitutes a Negative-Feedback Module Regulating Inflammatory Gene Expression. Mol. Cell 32, 371-382.

O'Leary, M.N., Schreiber, K.H., Zhang, Y., Duc, A.-C.E., Rao, S., Hale, J.S., Academia, E.C., Shah, S.R., Morton, J.F., Holstein, C.A., et al. (2013). The Ribosomal Protein Rpl22 Controls Ribosome Composition by Directly Repressing Expression of Its Own Paralog, Rpl22l1. PLoS Genet. 9, e1003708.

Parenteau, J., Durand, M., Morin, G., Gagnon, J., Lucier, J.-F., Wellinger, R.J., Chabot, B., and Abou Elela, S. (2011). Introns within Ribosomal Protein Genes Regulate the Production and Function of Yeast Ribosomes. Cell 147, 320-331.

Petibon, C., Parenteau, J., Catala, M., and Elela, S.A. (2016). Introns regulate the production of ribosomal proteins by modulating splicing of duplicated ribosomal protein genes. Nucleic Acids Res. 44, 3878-3891.

Plocik, A.M., and Guthrie, C. (2012). Diverse forms of RPS9 splicing are part of an evolving autoregulatory circuit. PLoS Genet 8, e1002620.

Raker, V.A., Mironov, A.A., Gelfand, M.S., and Pervouchine, D.D. (2009). Modulation of alternative splicing by long-range RNA structures in Drosophila. Nucleic Acids Res. 37, 4533-4544.

Rogic, S., Montpetit, B., Hoos, H.H., Mackworth, A.K., Ouellette, B.F., and Hieter, P. (2008). Correlation between the secondary structure of pre-mRNA introns and the efficiency of splicing in Saccharomyces cerevisiae. BMC Genomics 9, 355.

Rouskin, S., Zubradt, M., Washietl, S., Kellis, M., and Weissman, J.S. (2014). Genome-wide probing of RNA structure reveals active unfolding of mRNA structures in vivo. Nature 505, 701-705.

Schreiber, K., Csaba, G., Haslbeck, M., and Zimmer, R. (2015). Alternative Splicing in Next Generation Sequencing Data of Saccharomyces cerevisiae. PLoS ONE 10, e0140487.

Segev, N., and Gerst, J.E. (2018). Specialized ribosomes and specific ribosomal protein paralogs control translation of mitochondrial proteins. J. Cell Biol. 217, 117-126.

SenGupta, D.J., Zhang, B., Kraemer, B., Pochart, P., Fields, S., and Wickens, M. (1996). A three-hybrid system to detect RNA-protein interactions in vivo. Proc. Natl. Acad. Sci. 93, 8496-8501. 
Shi, Z., Fujii, K., Kovary, K.M., Genuth, N.R., Röst, H.L., Teruel, M.N., and Barna, M. (2017). Heterogeneous Ribosomes Preferentially Translate Distinct Subpools of mRNAs Genomewide. Mol. Cell 67, 71-83.e7.

Singh, N.N., Singh, R.N., and Androphy, E.J. (2006). Modulating role of RNA structure in alternative splicing of a critical exon in the spinal muscular atrophy genes. Nucleic Acids Res. 35, 371-389.

Spingola, M., and Ares, M. (2000). A Yeast Intronic Splicing Enhancer and Nam8p Are Required for Mer1p-Activated Splicing. Mol. Cell 6, 329-338.

Stumpf, C.R., Kimble, J., and Wickens, M. (2008). A Caenorhabditis elegans PUF protein family with distinct RNA binding specificity. RNA 14, 1550-1557.

Takagi, M., Absalon, M.J., McLure, K.G., and Kastan, M.B. (2005). Regulation of p53 Translation and Induction after DNA Damage by Ribosomal Protein L26 and Nucleolin. Cell 123, 49-63.

Vilardell, J., and Warner, J.R. (1994). Regulation of splicing at an intermediate step in the formation of the spliceosome. Genes Dev. 8, 211-220.

Vilardell, J., Chartrand, P., Singer, R.H., and Warner, J.R. (2000). The odyssey of a regulated transcript. RNA 6, 1773-1780.

Wan, Y., Kertesz, M., Spitale, R.C., Segal, E., and Chang, H.Y. (2011). Understanding the transcriptome through RNA structure. Nat. Rev. Genet. 12, 641-655.

Warner, J.R., and Mclntosh, K.B. (2009). How Common Are Extraribosomal Functions of Ribosomal Proteins? Mol. Cell 34, 3-11.

Yadav, A., Radhakrishnan, A., Panda, A., Singh, A., Sinha, H., and Bhanot, G. (2016). The Modular Adaptive Ribosome. PLOS ONE 11, e0166021.

Zhang, Y., Duc, A.-C.E., Rao, S., Sun, X.-L., Bilbee, A.N., Rhodes, M., Li, Q., Kappes, D.J., Rhodes, J., and Wiest, D.L. (2013). Control of Hematopoietic Stem Cell Emergence by Antagonistic Functions of Ribosomal Protein Paralogs. Dev. Cell 24, 411-425.

Zhang, Y., O'Leary, M.N., Peri, S., Wang, M., Zha, J., Melov, S., Kappes, D.J., Feng, Q., Rhodes, J., Amieux, P.S., et al. (2017). Ribosomal Proteins Rpl22 and Rpl22l1 Control Morphogenesis by Regulating Pre-mRNA Splicing. Cell Rep. 18, 545-556.

Zuker, M. (2003). Mfold web server for nucleic acid folding and hybridization prediction. Nucleic Acids Res. 31, 3406-3415. 


\section{Figure1}

A

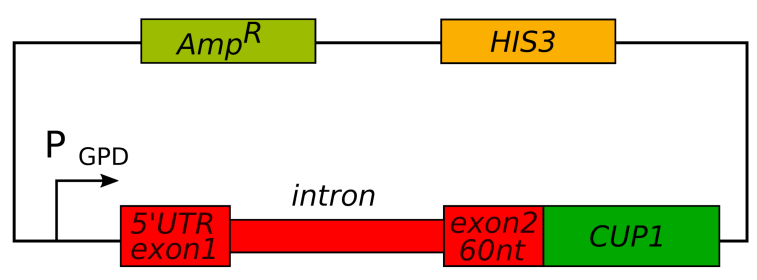

RPL22B reporter
B

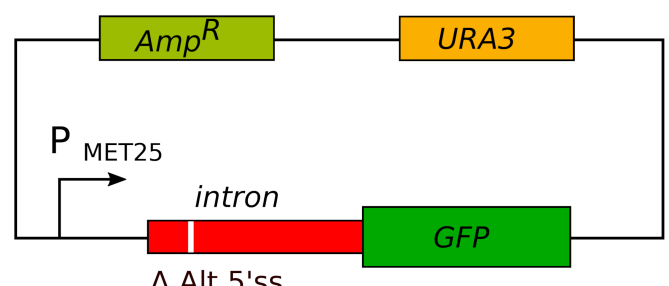

RPL22B reporter

\section{Fig. 1}

Comparison of reporter constructs used to analyze splicing of RPL22B in vivo.

(A) In this study, we used splicing reporter constructs based on p423GPD backbone with $R P L 22 B$ intron surrounded by its own 5'UTR, exon 1 and by 60 nts of exon 2 , which were fused in frame with CUP1 reporter (Abrhámová et al., 2018). Alternative 5'ss was preserved in all constructs unless indicated. (B) Gabunilas and Chanfreau used reporter plasmid derived from pUG23, where RPL22B intron without the surrounding sequences was inserted in front of the GFP reporter. In most of the constructs analyzed, alternative 5'ss was deleted (Gabunilas and Chanfreau, 2016). 


\section{Figure 2}

A

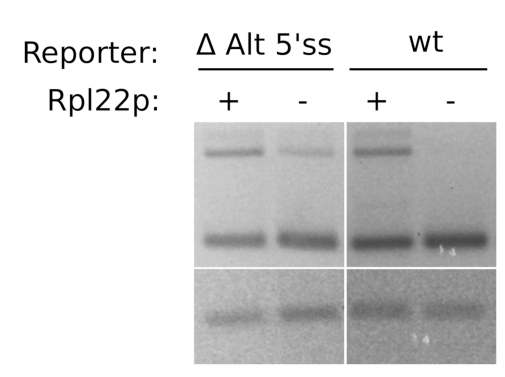

LDS

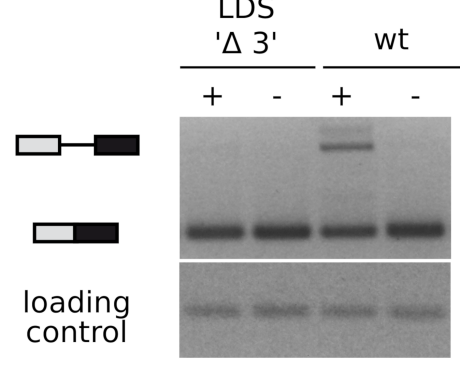

C

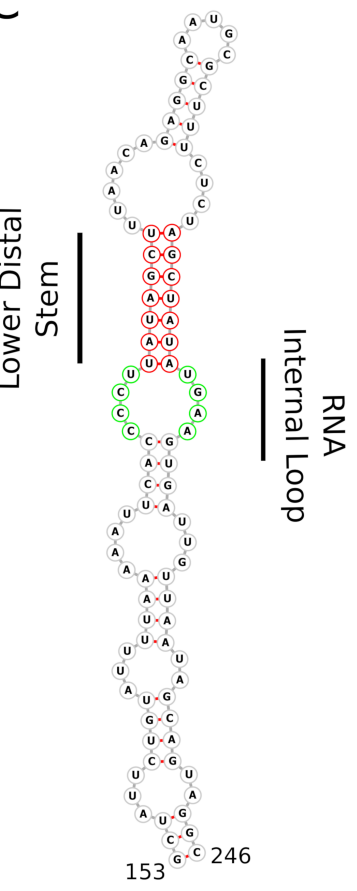

B

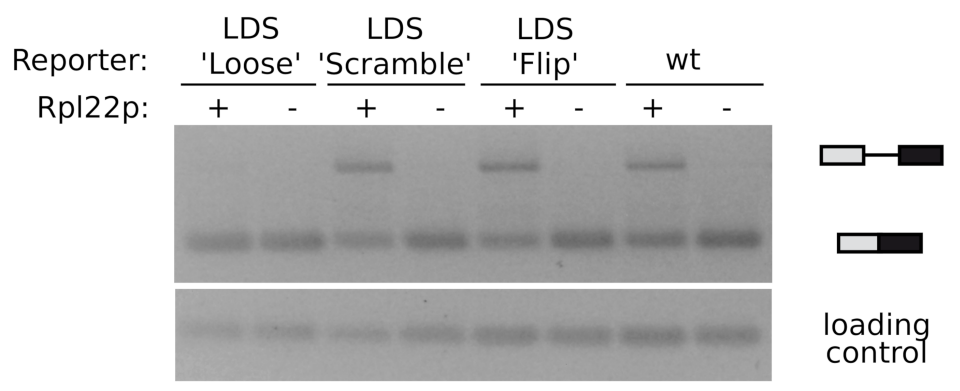

D

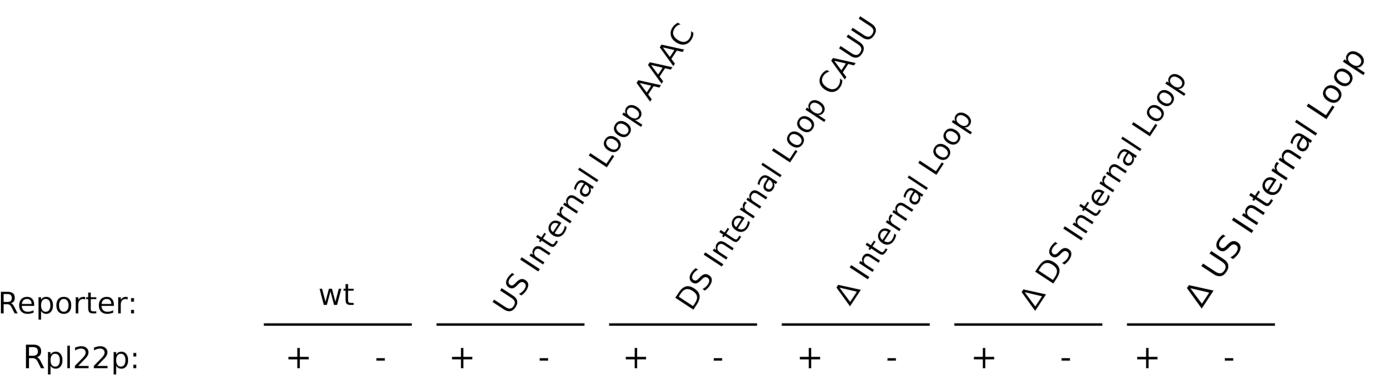

\section{Fig. 2}

Splicing analysis of RPL22B reporter constructs

Splicing efficiency of $R P L 22 B-C U P 1$ reporters was tested in $r p / 22 a \Delta r p / 22 b \Delta$ strain harboring pVTU260/RPL22A plasmid for Rpl22A overexpression and in the same strain transformed with empty vector. Semi-quantitative PCR was run on CDNA prepared using random hexamers from RNA isolated from exponentially growing cultures. SPT15 and SCR1 were used as loading control in $A, B$ and $D$, respectively. One of at least three independent 
experiments is shown. (A) Alternative 5'ss is required for efficient splicing. Deletion of alternative 5'ss increased the level of unspliced RNA in the absence of Rpl22 as compared to WT ( $\Delta$ Alt 5'ss). In comparison, deletion of 3' strand of the 'lower distal stem' (LDS) maintained efficient splicing but almost completely canceled splicing inhibition by Rpl22 (LDS ' $\Delta 3^{\prime}$ '). (B) Structure but not the sequence of LDS is important for splicing regulation. Mutants which maintained the pairing probabilities within the stem but changed its sequence ('Scramble', 'Flip') kept the regulation intact, but mutations that disrupted the structure of this part of the intron ('Loose' - mutation of nt 186GC to CG and 218AT to TA, ' $\triangle 3$ ' - deletion of nt 214-220 in A) lost the regulation. (C) Predicted secondary structure of $R P L 22 B i$ in a region implicated in splicing regulation (nt 153 - 246). Structure was predicted by RNA fold (Lorenz et al., 2011) and visualized using Forna (Kerpedjiev et al., 2015). LDS and 'RNA internal loop' are labelled red and green, respectively. (D) In contradiction to previous findings (Gabunilas and Chanfreau, 2016), nucleotides within the 'RNA internal loop' are dispensable for RPL22Bi splicing regulation. CCCU to AAAC mutation of upstream part of the loop ('US internal Loop AAAC'), UGAA to CAUU mutation in downstream part ('DS Internal Loop CAUU') as well as their deletions (deletion of both US CCCU and DS UGAA nucleotides - ' $\Delta$ Internal Loop', or per partes deletion of CCCU - ' $\Delta$ US Internal Loop' or UGAA - ' $\Delta$ DS Internal Loop') did not lose regulation upon Rpl22 protein overexpression. 


\section{Figure 3}

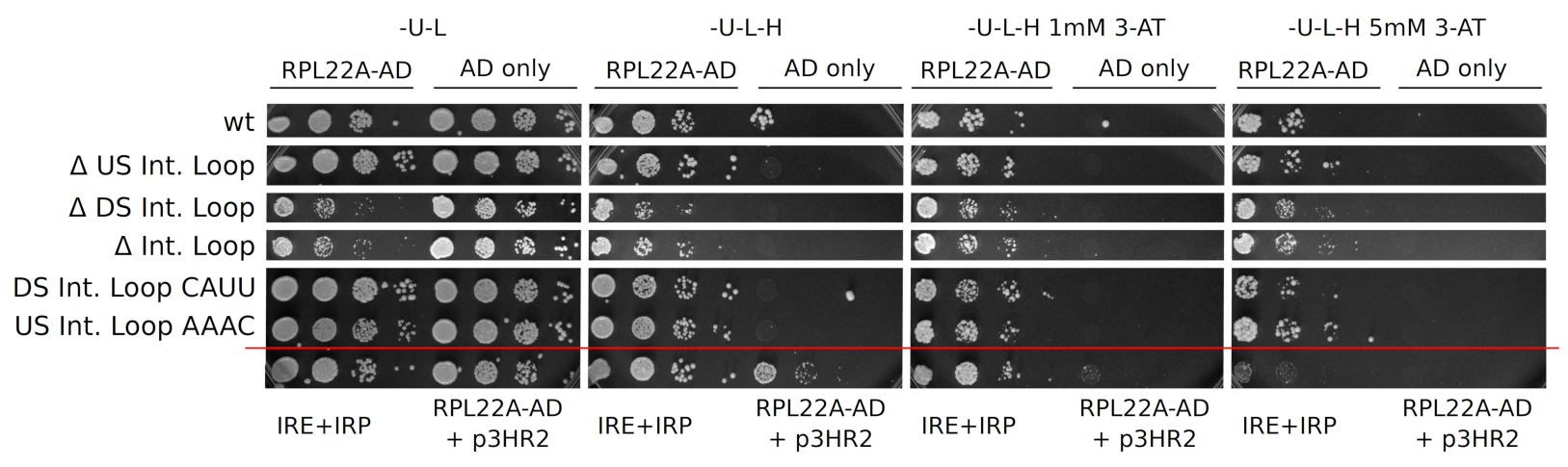

\section{Fig. 3}

Yeast three-hybrid analysis of Rpl22 binding to RPL22B intron

Previously, we reported that Rpl22 binds the intron of RPL22B between nt 165 and 236 (Abrhámová et al., 2018). We introduced 'RNA Internal Loop' mutations in this region (see text and Fig2) and tested them in yeast three-hybrid system for their ability to interact with Rpl22. The system uses HIS3 reporter gene, the activation of which depends on the recruitment of Rpl22 activation domain (AD) fusion protein through the interaction with the tested RNA. 10-fold serial dilutions of cells were spotted on plates with increasing concentrations of the metabolic retarder 3-aminotriazole (3-AT). '- $U$ ', ' $-L$ ', and '-H' denote the absence of uracil, leucine, and histidine in the medium. Cells with activation domain expressed alone (AD only, right parts of the panels) did not grow in the absence of histidine. Positive (IRE+IRP; SenGupta et al., 1996) and negative controls (Rpl22-AD with empty p3HR2 plasmid) are demarcated by red line (see also Abrhámová et al., 2018) for additional controls). Importantly, all RNA internal loop mutants supported cell growth to the same extent as the construct containing WT intron at 1 to 5 mM 3-AT. 